\title{
Diurnal preference, circadian phase of entrainment and time perspectives: Just what are the relationships?
}

\author{
Niall M. McGowan, Ross Brannigan, Darlene Doyle, Andrew N. Coogan * \\ Department of Psychology, Maynooth University, National University of Ireland, Maynooth, Co. Kildare, Ireland
}

\section{A R T I C L E I N F O}

\section{Article history:}

Received 28 October 2016

Received in revised form 15 February 2017

Accepted 22 February 2017

Available online 1 March 2017

\section{Keywords:}

Time perspective

Diurnal preference

Circadian

Chronotype

\begin{abstract}
A B S T R A C T
Time perspective refers to the non-conscious parcelling of personal and social experiences into temporal categories, and is an important construct in the psychology of time. Another important factor in time's influence on behavioural and psychological processes relates to the circadian timing system, which intrinsically produces daily rhythms in a host of parameters. This circadian timing system underpins timing of sleep/wake behaviour. Further, circadian timing is related to, but not synonymous with, diurnal preference for the timing of sleep/wake during different parts of the $24 \mathrm{~h}$ day. However, the exact nature of the relationship of diurnal preference to the underlying circadian clock is not elucidated. Previous research has indicated associations between time perspective and diurnal preference. In the current study we have examined the nature of the inter-relationships between circadian phase of entrainment (assessed with the Munich Chronotype Questionnaire), diurnal preference (assessed with the Morningness-Eveningness Questionnaire) and time perspectives in a sample of 193 adults. Both circadian phase and diurnal preference associate with present and future time perspectives, although when considered together in regression models, only diurnal preference predicts time preference. Further, we examined whether time perspectives might moderate the relationship between circadian phase of entrainment and diurnal preference, and find that this relationship is significantly moderated by present time perspectives, but not future time perspectives. These results indicate the intricate nature of interactions between domains of psychological and biological time.
\end{abstract}

(c) 2017 Elsevier Ltd. All rights reserved.

\section{Introduction}

Time is a dimension of immense psychological and physiological importance. Time perspective (TP), as defined by Stolarski, Bitner, and Zimbardo (2011), refers to the non-conscious parcelling of personal and social experiences into temporal categories. According to this concept, individuals differ in how they overemphasise aspects of the past, present, and future when making decisions and reflecting on events. Some people are more oriented towards the immediacy of the present whilst others are more sensitive to future outcomes and consequences. According to Zimbardo and Boyd (1999), time perspectives consist of dimensions relating to positive past, negative past, present hedonistic, present fatalistic and future. These time perspectives are found to be

Abbreviations: FTP, future time perspective; DBTP, deviation from a balanced time perspective; MEQ, Morningness-Eveningness Questionnaire; MCTQ Munich Chronotype Questionnaire; PTP, present time perspective; SJL, social jetlag; TP, time perspective; ZTPI, Zimbardo Time Perspective Inventory.

is The authors acknowledge funding to NMMcG from the John and Pat Hume Scholarship Scheme at Maynooth University.

* Corresponding author at: Department of Psychology, John Hume Building, Maynooth University, County Kildare, Ireland.

E-mail address: andrew.coogan@nuim.ie (A.N. Coogan). associated with several behavioural and psychological domains; for example, Diaz-Morales et al. (2007) report that low future orientation is associated with procrastination and indecision, Zimbardo, Keough, and Boyd (1997) report that present orientation is associated with risky driving, and Sailer et al. (2014) report that lower past negative and present fatalistic scores, coupled with higher present hedonistic scores, were associated with greater life satisfaction. Deviation from a balanced time perspective (Stolarski et al., 2011), describing the fit between an individual's TP and an optimal balance between past, present and future orientation, has previously been shown to be associated with poorer subjective well-being (Zhang, Howell, \& Stolarski, 2013), negative mood states (Stolarski, Matthews, Postek, Zimbardo, \& Bitner, 2014), and lower systemic cortisol production (Olivera-Figueroa, Juster, Morin-Major, Marin, \& Lupien, 2015).

In terms of biology, the temporal orchestration of behavioural control systems is of considerable importance. Evolution has conserved ancient daily time-keeping mechanisms which are present in virtually all eukaryotic organisms examined to date (Merrow, Spoelstra, \& Roenneberg, 2005). In mammals, a hierarchical network of endogenous circadian oscillators is responsible for the synchronisation of physiological and behavioural processes in response to, and in anticipation of, cyclical variations in the environment over a twenty-four hour period. 
Manifestation of circadian influences on the timing of behaviours can be ascertained from self-reported measures of actual, or preferred, sleep/ wake behaviour (Di Milia, Adan, Natale, \& Randler, 2013). The most commonly used instrument in this context is the MorningnessEveningness Questionnaire (MEQ; Horne \& Östberg, 1976) which produces a spectrum of preferred timing of sleep/wake behaviours (diurnal preference) as its output. Morningness refers to preferred earlier rise and bedtimes, whilst eveningness refers to preferred later rise and bedtimes. In humans there are considerable inter-individual, sex and ontological differences in diurnal preference (Adan et al., 2012). Another important instrument for assessing circadian influences on sleep/wake behaviours is the Munich Chronotype Questionnaire (MCTQ; Roenneberg, Wirz-Justice, \& Merrow, 2003). This instrument assesses actual timing of sleep/wake behaviours, rather than preferences for such as assessed by the MEQ, and uses the time of mid-sleep on free days (when masking psychosocial constraints on sleep timing are diminished) as a marker for the entrained circadian phase. Mid-sleep on free days closely tracks solar time, the principal time-cue to which circadian rhythms in humans entrain (Roenneberg, Kumar \& Merrow, 2007) and can be adjusted to control the confounds in measuring sleep phase associated with sleep debt experienced during the workweek (Roenneberg et al., 2004; Roenneberg, Kuehnle et al., 2007). It has been suggested that the MCTQ is a closer predictor of endogenous circadian phase than scores from the MEQ and there is a clear distinction in the measurement of sleep/wake behaviour versus psychological preference for such behaviour (Kantermann, Sung, \& Burgess, 2015; Levandovski, Sasso, \& Hidalgo, 2013; Roenneberg, 2015). Having said this, there is also concordance between MEQ and MCTQ scores (Zavada, Gordijn, Beersma, Daan, \& Roenneberg, 2005), and MEQ scores do relate to biological measures of circadian phase (Brown et al., 2008; Kantermann et al., 2015). However, the exact nature and extent of psychosocial and biological imperatives in shaping the timing of sleep/ wake behaviour remains unascertained, and the relationship between entrained circadian phase and diurnal preference is presently not clear.

Importantly, both domains of human temporality, TP and diurnal preference/entrained circadian phase, are predictors of several overlapping traits and behaviours. For example, studies examining TP have demonstrated that hedonistic and fatalistic present time perspectives are associated with greater risk-taking behaviour, poor impulse control, increased alcohol intake, smoking, and psychoactive drug use (Keough, Zimbardo, \& Boyd, 1999; Zimbardo \& Boyd, 1999), whilst future time perspective has been linked to conscientiousness, consideration for future consequences, and a preference for consistency (Adams \& Nettle, 2009; Zimbardo \& Boyd, 1999). Similarly, there have been findings of associations between eveningness and impulsivity, risk-taking, novelty and sensation seeking (Adan, Natale, Caci, \& Prat, 2010; Kilgore, 2007; Caci, Robert, \& Boyer, 2004; Ponzi, Wilson, \& Maestripieri, 2014), and morningness associating with conscientiousness, agreeableness, persistence, and cooperation with others (Diaz-Morales, 2007; Randler \& Saliger, 2011; Tsaousis, 2010).

Given the overlap in outcomes associated with both TP and the temporal organisation of daily behaviour, an intriguing question arises regarding the relation between the concept of psychological perspective of time and the underlying circadian time-keeping system. Some recent studies have attempted to examine this relationship. Stolarski, Ledzinska, and Matthews (2013) explored the relatedness of TP and diurnal preference on a sample of Polish university students and showed that that present time perspective (PTP) was associated with eveningness, whilst future time perspective (FTP) was related to morningness. These findings have since been replicated in cohorts from Germany and New Zealand (Nowack \& van der Meer, 2013; Milfont \& Schwazenthal, 2014). In the present study we aim to expand on these findings by examining TP associations with both diurnal preference (from the MEQ) and with estimates of entrained circadian phase (from the MCTQ). We hypothesise that due to the nature of the psychological preferences measured by the MEQ that diurnal preference would be more strongly associated with TP than mid-sleep on free days. Further, we hypothesise that the relationship between entrained circadian phase and diurnal preference may itself be influenced by preferences for different temporal frames, articulated as time perspectives. For example, individuals with a later phase of entrainment (as indicated with a later mid-sleep time) who are also present oriented may express later-than-expected diurnal preference (more eveningness) due to heavier discounting of potentially adverse future outcomes of late sleep-wake behaviour (e.g. shortened sleep, daytime sleepiness, high social jetlag), even if these preferences are not actualised as behaviours. As such, we set out to test the hypothesis that the relationship between entrained circadian phase (as measured by mid-sleep on the MCTQ) and diurnal preference would be moderated by time perspectives.

\section{Materials and methods}

\subsection{Participants}

A total sample of 193 participants ( 85 males and 108 females) were recruited via a convenience sampling method from the student population attending the host university or via an online survey administered to students $(n=97)$ and other members of the community-dwelling public $(n=96)$. The age range of the sample was between 18 and 64 with a mean age of $25.55(S D=9.47)$. Informed written consent was granted by each participant before commencing questionnaires or in the case of online presentation consent was agreed to electronically before proceeding to the test screen containing the questionnaires. The study protocol was approved by the Home University Research Ethics board and conformed to the Declaration of Helsinki.

\subsection{Measures}

\subsubsection{Morningness-Eveningness Questionnaire (MEQ).}

The Morningness-Eveningness Questionnaire (MEQ; Horne \& Östberg, 1976) was used to assess self-reported circadian typology in our sample. Responses on the 19-item scale were aggregated to produce an overall score with a range of 19-86. Low scores on the MEQ reflect a greater diurnal preference for eveningness whereas high scores at the other end of the continuum correspond with a greater orientation towards morningness. The Cronbach's $\alpha$ coefficient of the scale applied to the current sample was 0.87 .

\subsubsection{Munich Chronotype Questionnaire (MCTQ).}

The Munich Chronotype Questionnaire (MCTQ; Roenneberg et al., 2003) was used to measure the mid-point of sleep ('mid-sleep') of individuals which operates as an estimate of an individual's phase of entrainment. Respondents were asked to report their bedtimes and rise times on workdays and free days separately. The mid-point of sleep on free days corrected for sleep debt $\left(\mathrm{MSF}_{\mathrm{sc}}\right)$ was derived and used to measure chronotype (see Roenneberg et al., 2004). An estimate of recurring circadian misalignment or 'social jetlag' (SJL) was calculated by the difference between the mid-point of sleep on workdays and free days (Wittmann, Dinich, Merrow, \& Roenneberg, 2006; see supplement in Roenneberg, Allebrandt, Merrow, \& Vetter, 2012). MSF ${ }_{\mathrm{sc}}$ measured in local clock time, and SJL measured in hours were both decimalised (e.g. 3:45 becomes 3.75) for all analyses. MSF $_{\mathrm{sc}}$ was normally distributed as judged by Kolmogorov-Smirnov testing, SJL was not normally distributed.

\subsubsection{Zimbardo Time Perspective Inventory (ZTPI).}

Time perspective was measured using the Zimbardo Time Perspective Inventory (ZTPI; Zimbardo \& Boyd, 1999). The 56-item scale assesses each dimension of time perspective on a 5-point scale (ranging from $1=$ very untrue of me, $5=$ very true of me). The original ZTPI scoring procedure produces five factors measuring time perspective 
Table 1

Descriptive statistics for the variables of interest in this study. $n=85(44 \%)$ of participants were male and $n=108$ (56\%) were female.

\begin{tabular}{lll}
\hline & Mean & SD \\
\hline Age (Y) & 25.55 & 9.47 \\
MEQ & 46.12 & 10.47 \\
MSF $_{\text {SC }}($ h) & 5.34 & 1.27 \\
SJL (h) & 1.89 & 1.16 \\
PTP & 3.19 & 0.59 \\
FTP & 3.41 & 0.65 \\
DBTP & 5.82 & 2.85 \\
\hline
\end{tabular}

(TP): Past-Negative (PN) relates to a generally negative and unpleasant view of the past, Present-Hedonistic (PH) relates to a hedonistic and pleasure seeking attitude towards the here-and-now with little regard for future consequences, the Future (F) dimension relates to a general future orientation in an individual's decision making and planning, Past-Positive (PP) reflects a positive and sentimental view of the past, and Present-Fatalistic (PF) reveals a fatalistic perspective of the present and future life. Additionally the deviation from a balanced time perspective (DBTP) coefficient was derived from respondents' data from each of the original five ZTPI dimensions (see scoring algorithm in Stolarski et al., 2011). The DBTP coefficient measures the fit between an individual's time perspective profile and the 'ideal' optimal temporal perspective stated in Zimbardo and Boyd (2008). Values close to zero reflect a TP close to the theoretical ideal whereas greater coefficient values represent departures from a well-balanced TP.

It has previously been noted that many of the original ZTPI dimensions are confounded by positive or negative valences (Nowack, Milfont, \& van der Meer, 2013; Nowack \& van der Meer, 2013). For example, respondent endorsement on the PN item 'Painful past experiences keep being replayed in my mind' might be considered a measure of negative affect more so than an individual's bias towards a temporal perspective. Consequently we used ZTPI adapted scales that removed highly emotional items to produce a valence adjusted measure of time perspective which consisted of two factors, Present Time Perspective (PTP) and Future Time Perspective (FTP; see Nowack et al., 2013). This adjusted inventory consisted of 26 items ( 13 items for each factor).

\subsection{Data analysis}

We tested for normal distribution using Kolmogorov-Smirnov tests. Correlational analyses were conducted using Pearson $(r)$ or Spearman $\left(r_{r h o}\right)$ bivariate correlations as indicated for normally or non-normally distributed data respectively. Partial correlations controlling for age and sex were used where appropriate. For non-normally distributed data (SJL, DBTP, and FTP) partial Spearman correlations were conducted using the 'ppcor' package for R (Kim, 2015). All other analyses were conducted using SPSS 22 (IBM, Chicago, IL). For multiple regression analysis, variables were centred. Moderation analysis was conducted using the PROCESS macro for SPSS, utilising model 2 with bootstrapping with 1000 iterations (Hayes, 2012). Results are reported as significant where $p<0.05$. For moderation analysis $95 \%$ confidence intervals were also examined for exclusion of zero for interaction terms.

\section{Results}

The descriptive statistics for the variables of interest are presented in Table 1. Exploratory analysis revealed that age and sex were associated with MEQ scores, MSF ${ }_{\mathrm{sc}}$ and scores from the ZTPI. Consequently partial correlations controlling for the influence of age and sex were incorporated when examining the relation between diurnal preference/ entrained circadian phase and TP characteristics. All results from partial correlation analyses are presented in Table 2. Most noteworthy we report that higher MEQ scores (indicative of greater morningness) were positively associated with FTP (partial $r_{r h o}=0.246, p<0.001$, see Fig. 1A.) and inversely associated with PTP (partial $r=-0.285$, $p<0.001$, see Fig. 1B.). The same pattern was also found for the MSF ${ }_{\mathrm{sc}}$, with later mid-sleep being positively associated with PTP (partial $r=$ 0.229, $p<0.001$; Fig. 2) and inversely associated with FTP (partial $r_{r h o}=-0.170, p=0.003$; Fig. 2 ). Further, partial correlation analysis reveals that MEQ score associates with both PTP $\left(r_{r h o}=-0.205, p=\right.$ $0.004)$ and FTP $\left(r_{r h o}=240, p=0.001\right)$ when controlling for $\mathrm{MSF}_{\mathrm{sc}}$, but $\mathrm{MSF}_{\mathrm{sc}}$ does not significantly correlate with greater PTP $\left(r_{\text {rho }}=\right.$ $0.131, p=0.07)$ or lesser FTP $\left(r_{r h o}=-0.078, p=0.28\right)$ when controlling for MEQ score. SJL, the measure of discrepancy between mid-sleep on workdays and free days, was negatively associated with FTP (partial $r_{r h o}=-0.178, p=0.006$ ) but no associations were present between SJL and PTP. Interestingly SJL was associated with a greater DBTP coefficient score (partial $r_{r h o}=0.156, p=0.036$ ) but there was not any associations between DBTP and diurnal preference or $\mathrm{MSF}_{\mathrm{sc}}$.

In order to further probe the relationships between time perspectives and measures from the MEQ and MCTQ, we undertook 3 statistical forward linear regression analyses, with (1) FTP, (2) PTP and (3) DBTP as the dependent variables. The predictor variables inserted in each model were age, sex, MEQ score, $\mathrm{MSF}_{\mathrm{sc}}$ and SJL. The results are shown in Table 3. For PTP, MEQ was the only significant predictor and predicted $12 \%$ of the variance in PTP. For FTP, sex and MEQ were significant predictors, with MEQ predicting $12.6 \%$ of the variance in FTP. For DBTP, only SJL was a significant predictor (predicting $4.3 \%$ of the variance in DBTP).

Given that these results indicate a stronger association of MEQ score than $\mathrm{MSF}_{\mathrm{sc}}$ with time perspectives, we next analysed whether TP may moderate the relationship between entrained circadian phase $\left(\mathrm{MSF}_{\mathrm{Sc}}\right)$ and diurnal preference (MEQ score). We hypothesised that diurnal preference may emerge as a function of underlying circadian phase, but would also be moderated by other time-related psychological domains such as time perspectives. We conducted a moderation analysis of the relationship between $\mathrm{MSF}_{\mathrm{sc}}$ and MEQ, with PTP and FTP as moderators

Table 2

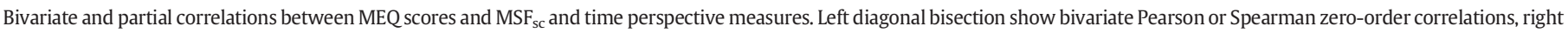
diagonal bisection (shaded) show correlations controlling for age and sex. ${ }^{*} p<0.05 ;{ }^{* *} p<0.01 ;{ }^{* * *} p<0.001$.

\begin{tabular}{|c|c|c|c|c|c|c|}
\hline & MEQ & MSFSC & SJL & PTP & FTP & DBTP \\
\hline MEQ & - & $-0.559^{* * *}$ & $-0.284^{* * *}$ & $-0.285^{* * *}$ & $0.246^{* * *}$ & -0.116 \\
\hline MSFSC & $-0.643^{* * *}$ & - & $0.417^{* * *}$ & $0.229^{* *}$ & $-0.170^{*}$ & 0.069 \\
\hline SJL & $-0.390^{* * *}$ & $0.529^{* * *}$ & - & 0.061 & $-0.178^{*}$ & $0.156^{*}$ \\
\hline PTP & $-0.356^{* * *}$ & $0.323^{* * *}$ & 0.167 & - & $-0.512^{* * *}$ & 0.181 \\
\hline FTP & $0.306^{* *}$ & $-0.260^{* * *}$ & $-0.241^{* *}$ & $-0.539^{* * *}$ & - & $-0.275^{* * *}$ \\
\hline DBTP & $-0.167^{*}$ & 0.140 & $0.206^{* *}$ & $0.217^{* *}$ & $-0.307^{* * *}$ & - \\
\hline
\end{tabular}


(Fig. 3). The overall $\mathrm{R}^{2}$ for the relationship of $\mathrm{MSF}_{\mathrm{sc}}$ and MEQ in this analysis was 0.493 . The results of the moderation analysis indicate that PTP significantly moderates the relationship between $\mathrm{MSF}_{\mathrm{sc}}$ and MEQ score $\left(p=0.0002\right.$ for interaction between $\mathrm{MSF}_{\mathrm{sc}}$ and PTP, $\left.\Delta \mathrm{R}^{2}=0.0397\right)$, whilst there was not significant moderation by FTP $\left(p=0.139 ; \Delta \mathrm{R}^{2}=0.006\right.$ ). Further analysis of the moderation of the relationship between MSF $_{\mathrm{sc}}$ and MEQ by PTP indicates that low PTP is associated with more eveningness arising from $\mathrm{MSF}_{\mathrm{sc}}$, whilst strong PTP is associated with less eveningness arising from $\mathrm{MSF}_{\mathrm{sc}}$ (Fig. 3). These results indicate that PTP may be an important factor in the relationship between underlying circadian phase and psychological diurnal preference.

\section{Discussion}

In this report we replicate findings from previous research linking eveningness with an increased orientation towards PTP, and morningness with an increased orientation towards FTP (Stolarski et al., 2013; Nowack \& van der Meer, 2013; Milfont \& Schwazenthal, 2014). Further, using $\mathrm{MSF}_{\mathrm{sc}}$ as a phase marker of the underlying entrainment of the circadian clock, we also describe similar correlations between later $\mathrm{MSF}_{\mathrm{sc}}$ and PTP, and earlier $\mathrm{MSF}_{\mathrm{sc}}$ and FTP. Possible explanations for these relationships between diurnal preference and timing of sleep/wake behaviour and time perspectives may involve a relationship between diurnal preference and temporal discounting (Milfont \& Schwazenthal, 2014), that result in preference for immediate reward of prolonging wake rather than going to bed. Resulting exposure to later evening light may in turn also further delay circadian phase (Duffy \& Czeisler, 2009). The current finding, that social jetlag is associated with unbalanced time perspective, could be reflective of this scenario in which time perspectives that deviate from the proposed ideal manifest themselves in unfavourable timing of sleep behaviour in the context of broader social imperatives (Wittmann et al., 2006). It is

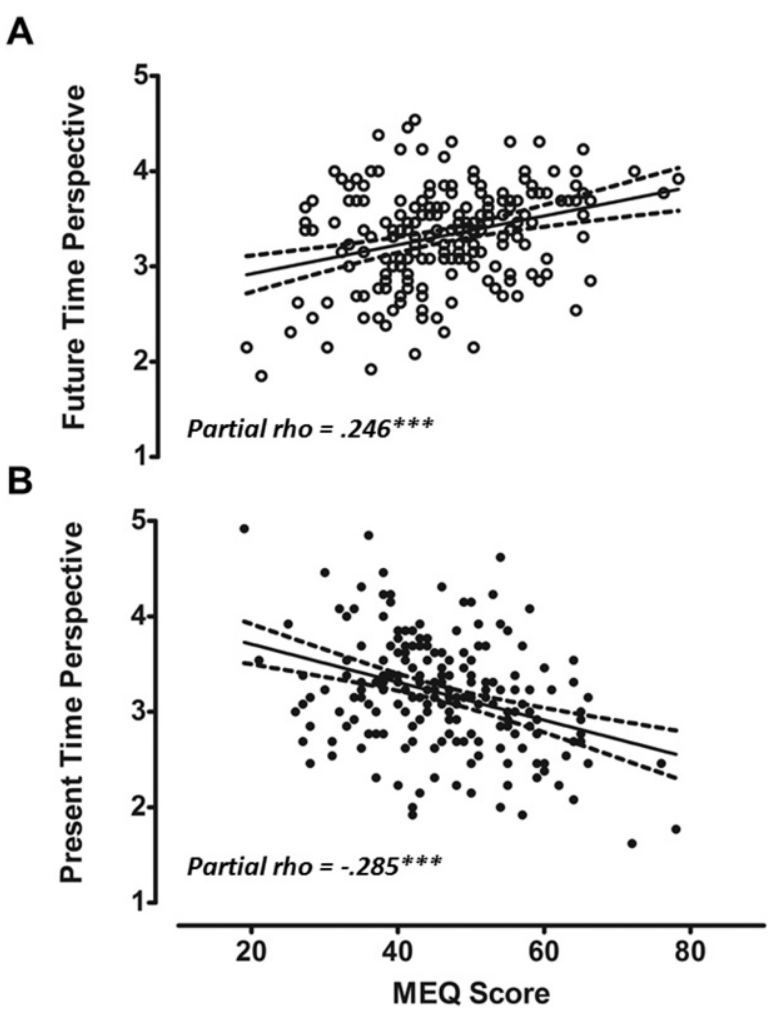

Fig. 1. Time perspectives associate with MEQ scores. Scatter-plots showing (A) positive relationship between MEQ scores (higher score, more morningness) and FTP (empty circles) and (B) inverse relationship between MEQ score and PTP (filled circles). ${ }^{* * *} p<0.001$.

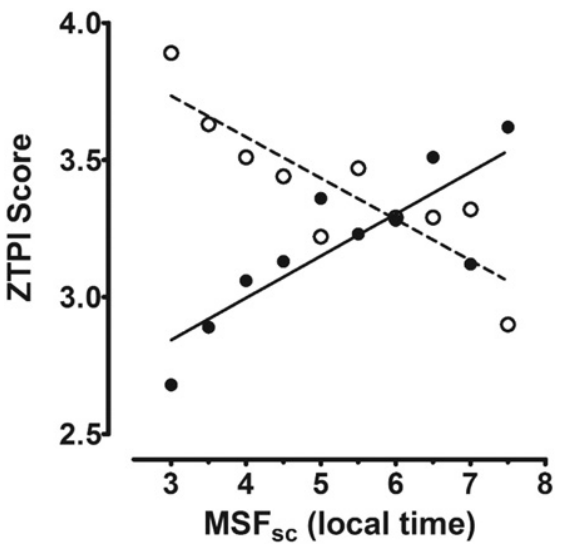

Fig. 2. Time perspectives associate with entrained circadian phase. Filled circles represent PTP score with the black line depicting positive relationship with $\mathrm{MSF}_{\mathrm{sc}}$, empty circles represent FTP score with broken line indicating negative relationship with MSF $_{\mathrm{sc}}$. Correlations based on unbinned raw data points, partial rho for $\mathrm{MSF}_{\mathrm{sc}}$ with PTP $=0.229$, $p<0.01$ and partial rho for $\mathrm{MSF}_{\mathrm{sc}}$ with FTP $=-0.170, p<0.05$.

interesting to note that despite our present findings of associations between PTP and FTP with MEQ and MCTQ scores, balanced time perspectives do not appear to have similar relationships with sleep/wake timing and preferences in our sample. One parsimonious explanation for this finding is that such relationships could be confounded by emotional valance (Nowack et al., 2013), as DBTP is calculated from the full 56-items of the ZTPI.

Our regression analysis indicates that MEQ score is a significant predictor of PTP and FTP when MSF $_{\mathrm{sc}}$ is included in the model, but the converse situation does not pertain. Therefore, time perspectives may be associated more with psychological aspects of diurnal preference in sleep/wake timing (as measured by the MEQ), rather than underlying circadian phase of entrainment. That a psychological preference, rather than a physiological state-marker, associates more strongly with the psychological construct of time perspective may not be overly surprising. Further, if diurnal preference is more strongly associated with time perspectives than entrained circadian phase, then it may be instructive to ask if time perspectives can illuminate the relationship between diurnal preference and $\mathrm{MSF}_{\mathrm{sc}}$. Roenneberg (2015) recently discussed the theoretical differences underpinning the development of the MEQ and the MCTQ pointing out that the MEQ derived from earlier work conceptualising diurnal preference as a dichotomous ('lark/owl') personality construct, whilst the MCTQ is based on circadian theory of entrainment. As such, the MEQ may be more suited towards the

Table 3

Results of forward statistical linear regression analysis with (A) PTP, (B) FTP and (C) DBTP as the dependent variables. The independent variables s entered in each of these analyses were MEQ score, $\mathrm{MSF}_{\mathrm{sc}}$, social jetlag (SJL), age and sex (coded as $0=$ male, $1=$ female).

\begin{tabular}{|c|c|c|}
\hline \multicolumn{3}{|c|}{ A } \\
\hline & & Step 0 (MEQ) \\
\hline $\begin{array}{l}\text { Model for predicting PTP score } \\
\text { MEQ }\end{array}$ & & $\begin{array}{l}\text { Adjusted } \mathrm{R}^{2}=0.122 \\
\beta=-0.356, p<0.001\end{array}$ \\
\hline \multicolumn{3}{|l|}{ B } \\
\hline & Step 0 (MEQ) & Step 1 (MEQ, sex) \\
\hline $\begin{array}{l}\text { Model for predicting FTP score } \\
\text { MEQ } \\
\text { Sex }\end{array}$ & $\begin{array}{l}\text { Adjusted } \mathrm{R}^{2}=0.126 \\
\beta=0.361, p<0.001\end{array}$ & $\begin{array}{l}\text { Adjusted } \mathrm{R}^{2}=0.151 \\
\beta=0.330, p<0.001 \\
\beta=-0.176, p=0.01\end{array}$ \\
\hline \multicolumn{3}{|l|}{ C } \\
\hline & & Step 0 (SJL) \\
\hline $\begin{array}{l}\text { Model for predicting DBTP score } \\
\text { SJL }\end{array}$ & & $\begin{array}{l}\text { Adjusted } \mathrm{R}^{2}=0.046 \\
\beta=-0.220, p=0.002\end{array}$ \\
\hline
\end{tabular}


A

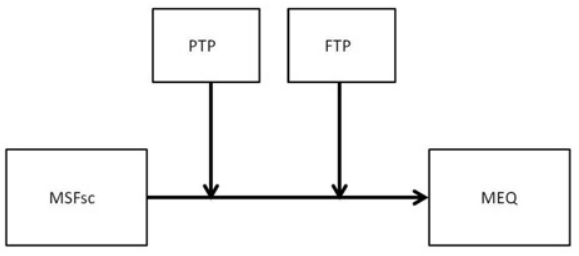

B

\begin{tabular}{lllllll}
\hline & Coefficient & SE & $\mathbf{t}$ & $\mathbf{P}$ & LLCl & ULCI \\
& & & & & & \\
\hline $\mathrm{MSF}_{\mathrm{SC}}$ & -4.95 & 0.46 & -10.03 & $<0.0001$ & -5.49 & -3.68 \\
\hline $\mathrm{PTP} \times \mathrm{MSF}_{\mathrm{SC}}$ & 3.19 & 0.83 & 3.83 & 0.002 & 1.53 & 4.83 \\
\hline FTP $\times \mathrm{MSF}_{\mathrm{SC}}$ & 1.18 & 0.709 & 1.48 & 0.14 & -0.39 & 2.74 \\
\hline
\end{tabular}

C

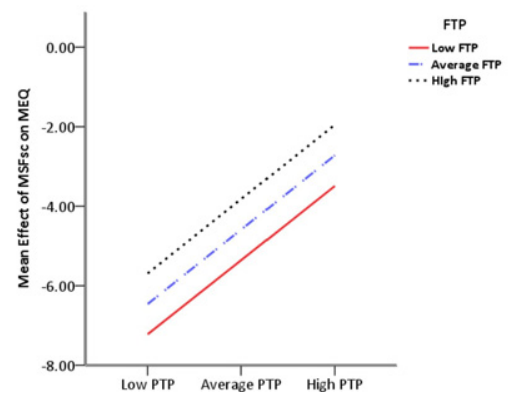

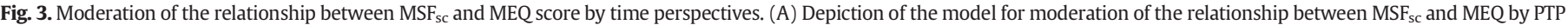

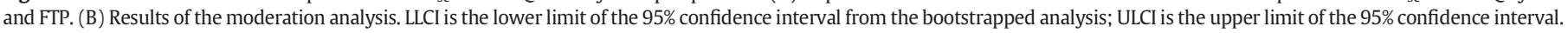

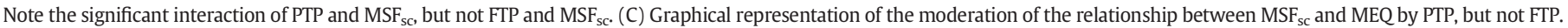

assessment of the relationships of diurnal preference with other psychological states and traits, whilst the MCTQ may be better suited to assessing physiological state of the clock in real-world conditions. However, MCTQ and MEQ measures strongly correlate in the current results and in other studies (Zavada et al., 2005) and MEQ scores also correlates with biological measures of clock function (Brown et al., 2008; Kantermann et al., 2015).

The essential nature of the relationship between preferred timing of sleep/wake activity and actual timing of sleep/wake behaviour is not well elucidated. Our moderation analysis indicates that time perspectives may impact this relationship, with lower levels of PTP associated with more eveningness emerging in relation to $\mathrm{MSF}_{\mathrm{sc}}$ scores. It is also interesting to note that although both PTP and FTP associate with diurnal preference and $\mathrm{MSF}_{\mathrm{sc}}$, only PTP moderates the relationship between MEQ score and $\mathrm{MSF}_{\mathrm{sc}}$. The reason for this directional moderation by PTP is not immediately clear; it may be that those with a lower PTP have greater psychosocial constraints on their sleep/wake behaviour, and so the disparity between preferred timing and actual timing of sleep is more accentuated (Di Milia et al., 2013). Alternatively, it may be that specific items and domains assessed in the MEQ (which are not reflected in the calculation of $\mathrm{MSF}_{\mathrm{sc}}$ from the MCTQ) may be differentially influenced by time perspectives. For example, the MEQ has items relating to alertness, hunger and exercise, representing factors that could be influenced by present time perspective but which may not be reflected in $\mathrm{MSF}_{\mathrm{sc}}$ scores. Another important consideration involves personality domains; it is reported that both earlier diurnal preference and future time perspective are associated with conscientiousness (Kairys, 2015; Tonetti, Fabbri, \& Natale, 2009) whilst later diurnal preference and present time perspectives are associated with impulsivity, sensation seeking, sociability and neuroticism (McGowan, Voinescu, \& Coogan, 2016; Duggan, Friedman, McDevitt, \& Mednick, 2014; Muro et al., 2015). Future work should seek to address carefully these factors in advancing our understanding of the psychological determinants of sleep/wake behaviour timing.

In conclusion, time perspectives may be important factors in moderating the emergence of diurnal preference from the biological construct of underlying circadian phase of entrainment. The current findings point to the importance of identifying psychological factors that shape diurnal preference in concert with the endogenous circadian clock, and highlight the possible interactions of all psychological aspects time in shaping human behaviour.

\section{Conflict of interest}

The authors declare no conflict of interest.

\section{References}

Adams, J., \& Nettle, D. (2009). Time perspective, personality and smoking, body mass, and physical activity: An empirical study. British Journal of Health Psychology, 14, 83-105.

Adan, A., Archer, S. N., Hidalgo, M. P., Di Milia, L., Natale, V., \& Randler, C. (2012). Circadian typology: A comprehensive review. Chronobiology International, 29(2), 1153-1175.

Adan, A., Natale, V., Caci, H., \& Prat, G. (2010). Relationship between circadian typology and functional and dysfunctional impulsivity. Chronobiology International, 27(3), 606-619.

Brown, S. A., Kunz, D., Dumas, A., Westermark, P. O., Vanselow, K., Tilmann-Wahnschaffe, A., ... Kramer, A. (2008). Molecular insights into human daily behavior. Proceedings of the National Academy of Science USA, 105(5), 1602-1607.

Caci, H., Robert, P., \& Boyer, P. (2004). Novelty seekers and impulsive subjects are low in morningness. European Journal of Psychiatry, 19, 79-84

Di Milia, L., Adan, A., Natale, V., \& Randler, C. (2013). Reviewing the psychometric properties of contemporary circadian typology measures. Chronobiology International, 30(10), 1261-1271.

Diaz-Morales, J. F. (2007). Morning and evening-types: Exploring their personality styles. Personality and Individual Differences, 43, 769-778.

Duffy, J. F., \& Czeisler, C. A. (2009). Effect of light on human circadian physiology. Sleep Medicine Clinics, 4(2), 165-177. 
Duggan, K. A., Friedman, H. S., McDevitt, E. A., \& Mednick, S. C. (2014). Personality and healthy sleep: The importance of conscientiousness and neuroticism. PloS One, 9(3), e90628.

Hayes, A. F. (2012). PROCESS: A versatile computational tool for observed variable mediation, moderation, and conditional process modeling [White paper]. Retrieved from http://www.afhayes.com/public/process2012.pdf

Horne, J., \& Östberg, O. (1976). A self-assessment questionnaire to determine morningness eveningness in human circadian rhythms. International Journal of Chronobiology, 4, 97-110.

Kairys, A. (2015). Time perspective and personality. In Stolarski, Fieulaine, \& van Beek (Eds.), Time perspective theory; Review, research and application. Springer.

Kantermann, T., Sung, H., \& Burgess, H. J. (2015). Comparing the MorningnessEveningness Questionnaire and Munich Chronotype Questionnaire to the dim light melatonin onset. Journal of Biological Rhythms, 30(5), 449-453.

Keough, K. A., Zimbardo, P. G., \& Boyd, J. N. (1999). Who's smoking, drinking, and using drugs? Time perspective as a predictor of substance use. Basic and Applied Social Psychology, 21(2), 149-164.

Kilgore, W. D. (2007). Effects of sleep deprivation and morningness-eveningness traits on risk-taking. Psychology Reports, 100(2), 613-626.

Kim, S. (2015). Ppcor: An R package for a fast calculation to semi-partial correlation coefficients. Communications for Statistical Applications and Methods, 22(6), 665-674.

Levandovski, R., Sasso, E. \& Hidalgo, M. P. (2013). Chronotype: A review of the advances, limits and applicability of the main instruments used in the literature to assess human phenotype. Trends in Psychiatry and Psychotherapy, 35, 3-11.

McGowan, N. M., Voinescu, B. I., \& Coogan, A. N. (2016). Sleep quality, chronotype and social jetlag differentially associate with symptoms of attention deficit hyperactivity disorder in adults. Chronobiology International, 33(10), 1433-1443.

Merrow, M., Spoelstra, K., \& Roenneberg, T. (2005). The circadian cycle: Daily rhythms from behaviour to genes. EMBO Reports, 6, 930-935.

Milfont, T. L., \& Schwazenthal, M. (2014). Explaining why larks are future-oriented and owls are present-oriented: Self-control mediates the chronotype-time perspective relationships. Chronobiology International, 31(4), 581-588.

Muro, A., Castella, J., Sotoca, C., Estaun, S., Valero, S., \& Goma-i-Freixanet, M. (2015). To what extent is personality associated with time perspective? Anales De Psychologica, 31(2), 488-493.

Nowack, K., Milfont, T., \& van der Meer, E. (2013). Future versus present: Time perspective and pupillary response in a relatedness judgement task investigating temporal event knowledge. International Journal of Psychophysiology, 87, 173-182.

Nowack, K., \& van der Meer, E. (2013). Are larks future-oriented and owls present-oriented? Age- and sex-related shifts in chronotype-time perspective associations. Chronobiology International, 30(10), 1240-1250.

Olivera-Figueroa, L. A., Juster, R. -P., Morin-Major, J. K., Marin, M. -F., \& Lupien, S. J. (2015). A time to be stressed? Time perspectives and cortisol dynamics among healthy adults. Biological Psychology, 111, 90-99.

Ponzi, D., Wilson, M. C., \& Maestripieri, D. (2014). Eveningness is associated with higher risk-taking, independent of sex and personality. Psychological Reports, 115(3), 932-947.
Randler, C., \& Saliger, L. (2011). Relationship between morningness-eveningness and temperament and character dimensions in adolescents. Personality and Individual Differences, 50(2), 148-152.

Roenneberg, T. (2015). Having trouble typing? What on Earth is chronotype? Journal of Biological Rhythms, 30(6), 487-491.

Roenneberg, T., Allebrandt, K. V., Merrow, M., \& Vetter, C. (2012). Social jetlag and obesity. Current Biology, 22(10), 939-943.

Roenneberg, T., Kuehnle, T., Juda, M., Kantermann, T., Allebrandt, K., Gordijn, M., \& Merrow, M. (2007a). Epidemiology of the human circadian clock. Sleep Medicine Reviews, 11(6), 429-438.

Roenneberg, T., Kuehnle, T., Pramstaller, P. P., Ricken, J., Havel, M., Guth, A., \& Merrow, M. (2004). A marker for the end of adolescence. Current Biology, 14(24), R1038.

Roenneberg, T., Kumar, C. J., \& Merrow, M. (2007b). The human circadian clock entrains to sun time. Current Biology, 17, R44-R45.

Roenneberg, T., Wirz-Justice, A., \& Merrow, M. (2003). Life between clocks: Daily temporal patterns of human chronotypes. Journal of Biological Rhythms, 18(1), 80-90.

Sailer, U., Rosenberg, P., Nima, A. A., Gamble, A., Gärling, T., Archer, T., \& Garcia, D. (2014) A happier and less sinister past, a more hedonistic and less fatalistic present and a more structured future: Time perspective and well-being. PeerJ, 2, e303.

Stolarski, M., Bitner, J., \& Zimbardo, P. G. (2011). Time perspective, emotional intelligence and discounting of delayed awards. Time and Society, 20(3), 346-363.

Stolarski, M., Ledzinska, M., \& Matthews, G. (2013). Morning is tomorrow, evening is today: Relationships between chronotype and time perspective. Biological Rhythm Research, 44, 181-196.

Stolarski, M., Matthews, G., Postek, S., Zimbardo, P. G., \& Bitner, J. (2014). How we feel is a matter of time: Relationships between time perspectives and mood. Journal of Happiness Studies, 15(4), 809-827.

Tonetti, L., Fabbri, M., \& Natale, V. (2009). Relationship between circadian typology and big five personality domains. Chronobiology International, 26(2), 337-347.

Tsaousis, I. (2010). Circadian preferences and personality traits: A meta-analysis. European Journal of Personality, 24, 356-373.

Wittmann, M., Dinich, J., Merrow, M., \& Roenneberg, T. (2006). Social jetlag: misalignment of biological and social time. Chronobiology International, 23(1\&2), 497-509.

Zavada, A., Gordijn, M. C., Beersma, D. G., Daan, S., \& Roenneberg, T. (2005). Comparison of the Munich Chronotype Questionnaire with the Horne-Ostberg's morningnesseveningness score. Chronobiology International, 22(2), 267-278.

Zhang, J. W., Howell, R. T., \& Stolarski, M. (2013). Comparing three methods to measure a balanced time perspective: The relationship between a balanced time perspective and subjective well-being. Journal of Happiness Studies, 14, 169-184.

Zimbardo, P. G., \& Boyd, J. N. (1999). Putting time in perspective: A valid, reliable individual-differences metric. Journal of Personality and Social Psychology, 77, 1271-1281.

Zimbardo, P. G., \& Boyd, J. N. (2008). The time paradox. New York, NY: Free Press.

Zimbardo, P. G., Keough, K. A., \& Boyd, J. N. (1997). Present time perspective as a predictor of risky driving. Personality and Individual Differences, 23(6), 1007-1023. 